\title{
A Robust Fault-Tolerant Control for Quadrotor Helicopters against Sensor Faults and External Disturbances
}

\author{
Ban Wang $\mathbb{D}^{1}{ }^{1}$ Peng Huang, ${ }^{2}$ and Wei Zhang ${ }^{1}$ \\ ${ }^{1}$ School of Aeronautics, Northwestern Polytechnical University, Xi'an, Shaanxi 710072, China \\ ${ }^{2}$ Chinese Flight Test Establishment, Xi'an, Shaanxi 710089, China \\ Correspondence should be addressed to Ban Wang; wangban@nwpu.edu.cn
}

Received 2 January 2021; Revised 2 February 2021; Accepted 6 March 2021; Published 20 March 2021

Academic Editor: Jing $\mathrm{Na}$

Copyright (c) 2021 Ban Wang et al. This is an open access article distributed under the Creative Commons Attribution License, which permits unrestricted use, distribution, and reproduction in any medium, provided the original work is properly cited.

\begin{abstract}
This paper presents an active fault-tolerant control strategy for quadrotor helicopters to simultaneously accommodate sensor faults and external disturbances. Unlike most of the existing fault diagnosis and fault-tolerant control schemes for quadrotor helicopters, the proposed fault diagnosis scheme is able to estimate sensor faults while eliminating the effect of external disturbances. Moreover, the proposed fault-tolerant control scheme is capable to eliminate the adverse effect of external disturbances as well by designing a disturbance observer to effectively estimate the unknown external disturbances and integrating with the designed integral sliding-mode controller. In this case, the continuous operation of the quadrotor helicopter is ensured while avoiding the unexpected control chattering. In addition, the stability of the closed-loop system is theoretically proved. Finally, the effectiveness and advantages of the proposed scheme are validated and demonstrated through comparative numerical simulations of the quadrotor helicopter under different faulty and uncertain scenarios.
\end{abstract}

\section{Introduction}

Unmanned quadrotor helicopters have recently been widely used by commercial industries and academic communities due to their vertical take-off and landing capability, low cost, and great maneuverability. In order to allow the aircraft to track desired orientation commands, while preventing it from flipping over and crashing, the quadrotor attitude control system is becoming more and more sophisticated with increasing requirements for high performance and reliability. Many control approaches have been proposed for tracking control of quadrotor helicopters [1-3]. However, most of the aforementioned literatures did not take potential faults in the system into account. Sensors are identified as the weak link in aerospace engineering systems based on being more vulnerable to damage or being more sensitive in construction than other components $[4,5]$. A fault in an attitude sensor can be regarded as an unexpected change of sensor operation affecting the sensor output $[6,7]$. In the case of sensor faults, measurements of state variables and other quantities of interests may no longer be reliable which may degrade quadrotor flight performance or even cause a catastrophic accident. Thus, the faulty measurements cannot be used for output or state feedback control directly without any correction algorithms. To meet the stringent demands of system reliability, physical redundancy in the form of identical sensors or multiple types of sensors is often configured [8]. Nevertheless, redundant sensors are not always feasible due to the constraints of cost, weight, and complexity, especially for small-size unmanned aerial vehicles (UAVs). In this case, analytical redundancy constituted by the knowledge of the considered system becomes a viable supplement. In the study by Zhong et al. [9], a two-stage extended Kalman filter is developed for detecting, isolating, and identifying inertial measurement unit (IMU) sensor faults of an unmanned quadrotor helicopter, while mitigating the impact of model uncertainties. In the study by Matus-Vargas et al. [10], a fault-tolerant control scheme for a multirotor with altitude sensor faults caused by the ground effect is presented and validated by simulations and experiments. In the study by Zhang and Xiao [11], a sliding mode observer with the adaptive regulation law is proposed 
for multiple sensor fault detection and reconstruction without knowing the upper bound of the unknown faults. In the study by Tan et al. [12], a sliding mode control combined with the RBF neural network is designed to deal with the failures of the sensor and actuator. Considerable research results have been reported on sensor fault diagnosis. A fault diagnosis system normally performs three major tasks: fault detection, fault isolation, and fault estimation. Among various types of fault diagnosis approaches that have been developed, sliding mode observer-based fault diagnosis has been proven to be an effective way to detect, isolate, and estimate faults for many systems due to the inherent robustness of sliding mode algorithms to unknown modeling uncertainties and disturbances [13-15]. In the study by Patton et al. [13], the derivative of the considered sensor fault is assumed to be zero, and the concept of equivalent output injection is employed to reconstruct the fault. Then, in the study by Tan and Edwards [14], the assumption of constant sensor fault is relaxed to a more general case but it assumes that the considered system needs to be open-loop stable. In the study by Alwi et al. [15], the proposed scheme overcomes the limitation that the open-loop system is required to be stable and is capable to estimate incipient sensor faults. Note that, the aforementioned research only deals with the diagnosis of sensor faults. From the control point of view, after fault occurrence, whether the fault can be compensated and the system can maintain its original tracking performance are of importance. In other words, the integrated design of the controller and fault diagnosis scheme needs to be further investigated. The stability of the closed-loop system with reconstructed measurements needs to be proved. Therefore, with application to quadrotor helicopters, the fault diagnosis and fault-tolerant control for quadrotor helicopters has been an important research topic in recent years with the growing demands for safety, reliability, and maintainability [16-18]. The timely diagnosis and correction of faults in a quadrotor system are critical in avoiding abnormal system performance and reducing the unexpected accidents significantly.

During the last decade, various fault diagnosis approaches have been proposed with the advances in control theory [19-23]. Most of the proposed diagnosis systems contain only fault detection and isolation (FDI), which cannot provide more comprehensive information of the corresponding fault, such as magnitude of the fault. Fault estimation, which is also referred to as fault identification, can provide the estimated magnitude of the fault. It plays an important role in active fault-tolerant control (AFTC) since it can provide the required fault information to reconfigure the controller. In [24, 25], nonlinear adaptive estimation methods are presented to detect, isolate, and estimate sensor bias faults in accelerometer and gyroscope measurements of a quadrotor helicopter. In the study by Zhong et al. [26], an adaptive two-stage extended Kalman filter is proposed for sensor fault detection and diagnosis of a quadrotor helicopter based on its kinematic model. Nonetheless, fault diagnosis is a very important procedure, and it is not sufficient to ensure safe operation of the quadrotor helicopter. For safety-critical systems, the diagnosis of a particular system fault is not the ultimate goal, whereas the continuity of operation is a key feature, and the closed-loop system should be capable of maintaining its prespecified performance in terms of quality, safety, and stability despite the presence of faults [27]. This calls for the appearance of FTC systems.

In the context of FTC for quadrotor helicopters, most of the research works in the literature focus on actuator faults [28-32]. Research on sensor FTC for quadrotor helicopters is very limited compared to the sensor fault diagnosis system and actuator FTC system $[33,34]$. In the study by Zhang et al. [33], a tracking controller and a sensor fault detection and isolation scheme were separately developed for a quadrotor helicopter. The proposed fault diagnosis system can only detect and isolate sensor faults and is not integrated with the designed controller to accommodate faults in an active fashion. In the study by Qin et al. [34], a fault diagnosis unit is designed to estimate sensor faults and synthesized with a two-level PID controller to constitute an active FTC scheme for a quadrotor helicopter. However, the proposed scheme in [34] is based on a linear model of the quadrotor helicopter. In practical situations, the existence of system parameter variations, nonlinearity, and disturbances can make the model-based fault diagnosis and FTC system ineffective. Consequently, it is vital to take robustness into consideration, while designing model-based fault diagnosis and FTC systems.

In an attempt to solve the aforementioned difficulties for improving system robustness, this paper presents a novel observer-based fault diagnosis and FTC scheme for quadrotor helicopters against sensor faults and disturbances. The main contributions of this paper are summarized as follows:

(1) In the face of sensor faults and external disturbances, an active FTC scheme is proposed to simultaneously accommodate them in terms of both fault diagnosis and fault-tolerant control based on integral slidingmode control, sliding mode observer, and disturbance observer.

(2) Unlike most of the existing fault diagnosis schemes for quadrotor helicopters, the proposed fault diagnosis scheme is able to estimate sensor faults, while eliminating the effect of external disturbances.

(3) A disturbance observer is constructed to estimate the lumped disturbances and integrated with the designed sliding-mode controller, which can help to avoid the unexpected control chattering. The stability of the closed-loop system is theoretically proved.

The rest of this paper is arranged as follows. The dynamic model of the studied quadrotor helicopter and problem statement are presented in Section 2. The detailed design procedures of the proposed fault diagnosis and FTC scheme are described in Section 3. Then, the numerical simulation results are demonstrated in Section 4 to validate the effectiveness of the proposed scheme. Finally, general conclusions of this paper are summarized in Section 5 . 


\section{Quadrotor Model and Problem Statement}

2.1. Quadrotor Model Description. The studied quadrotor helicopter is an underactuated system with six degrees of freedom driven by four motors mounted in a cross configuration, as shown in Figure 1. The voltage inputs imposed on the motors drive the corresponding propellers to generate forces and torques. Each pair of the opposite propellers turns the same way, i.e., the front and rear propellers rotate clockwise and the left and right ones spin counterclockwise. In order to facilitate the modeling of the quadrotor helicopter, two coordinate systems are employed: the earth-fixed frame $\{E\}$ and the body-fixed frame $\{B\}$. The origin of the earth-fixed frame is attached on the ground, while the origin of the body-fixed frame is coincident with the center of gravity of the studied quadrotor helicopter. The axes of the earth-fixed frame are denoted as $\left(o_{e}, x_{e}, y_{e}, z_{e}\right)$, and the axes of the body-fixed frame are denoted as $\left(o_{b}, x_{b}, y_{b}, z_{b}\right)$.

Considering the attitude control of the quadrotor helicopter, by employing the Newton-Euler formulation, the dynamic model of the quadrotor helicopter can be expressed as follows:

$$
\tau^{B}=J \dot{\omega}^{B}+\omega^{B} \times J \omega^{B},
$$

where $\tau^{B}=\left[\tau_{x}, \tau_{y}, \tau_{z}\right]^{T}$ is the resultant torque vector and $\omega^{B}=[p, q, r]^{T}$ is the angular velocity vector with respect to the body-fixed frame, respectively, and $J=$ diag $\left(\left[J_{x}, J_{y}, J_{z}\right]\right)=\operatorname{diag}([0.03,0.03,0.04]) \mathrm{kg} \cdot \mathrm{m}^{2}$ is a diagonal matrix that stands for the moments of inertia of three axes of the studied quadrotor helicopter with the assumption that the axes of the body-fixed frame are coincident with the principal axes of inertia of the studied quadrotor helicopter.

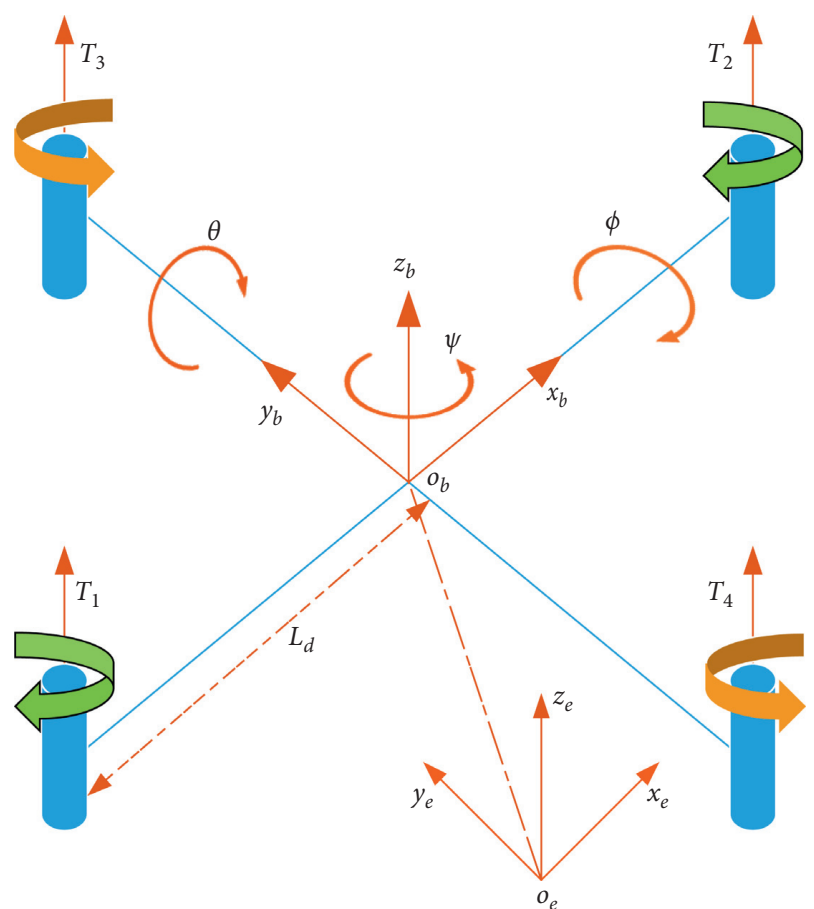

FIgURE 1: The configuration of the studied quadrotor helicopter.

The corresponding torques imposed on the quadrotor helicopter consist of the propeller-generated torque, the gyroscopic torque, and the rotational motion-induced torque, which can be described as

$$
\tau^{B}=\left[\begin{array}{cccc}
0 & 0 & K_{u} L_{d} & -K_{u} L_{d} \\
K_{u} L_{d} & -K_{u} L_{d} & 0 & 0 \\
K_{y} & K_{y} & -K_{y} & -K_{y}
\end{array}\right]\left[\begin{array}{l}
u_{1} \\
u_{2} \\
u_{3} \\
u_{4}
\end{array}\right]-\sum_{i=1}^{4} I_{r}\left(\omega^{B} \times\left[\begin{array}{l}
0 \\
0 \\
1
\end{array}\right]\right)(-1)^{i+1} \Omega_{i}+\left[\begin{array}{c}
-k_{d 1} \dot{\phi} \\
-k_{d 2} \dot{\theta} \\
-k_{d 3} \dot{\psi}
\end{array}\right],
$$

where $\phi, \theta$, and $\psi$ is the roll, pitch, and yaw angle of the quadrotor helicopter defined in the earth-fixed frame, respectively, $I_{r}$ is the inertial moment of the propeller, $\Omega_{i}(i=$ $1,2, \ldots, 4)$ is the $i$ th propeller's rotational speed, $k_{d i}(i=$ $1,2, \ldots, 4)$ is the drag coefficient, and $u_{i}(i=1,2, \ldots, 4)$ is the pulse width modulation (PWM) input of the $i$ th motor, and the control input signals are within the range $[0.05,0.1]$. A command of 0.05 corresponds to zero throttle, which will cause the motors to stop, and a command of 0.1 corresponds to full throttle. $K_{u}=120 \mathrm{~N}$ is a positive gain related to propeller-generated force, $K_{y}=4 \mathrm{Nm}$ is a positive gain related to propeller-generated torque, and $L_{d}=0.2 \mathrm{~m}$ is the distance between the motor and the center of gravity of the quadrotor helicopter.
Then, by substituting (2) into (1), it leads to

$$
\left\{\begin{array}{l}
\dot{p}=\frac{J_{y}-J_{z}}{J_{x}} q r+\frac{u_{\phi}}{J_{x}}-I_{r} \dot{\theta} \Omega-k_{d 1} \dot{\phi}, \\
\dot{q}=\frac{J_{z}-J_{x}}{J_{y}} p r+\frac{u_{\theta}}{J_{y}}+I_{r} \dot{\phi} \Omega-k_{d 2} \dot{\theta}, \\
\dot{r}=\frac{J_{x}-J_{y}}{J_{z}} p q+\frac{u_{\psi}}{J_{z}}-k_{d 3} \dot{\psi},
\end{array}\right.
$$

where $u_{\phi}=K_{u} L_{d}\left(u_{3}-u_{4}\right), u_{\theta}=K_{u} L_{d}\left(u_{1}-u_{2}\right)$, and $u_{\psi}=$ $K_{y}\left(u_{1}+u_{2}-u_{3}-u_{4}\right)$. 
The kinematic differential equation of the quadrotor helicopter can be described as

$$
\left[\begin{array}{l}
\dot{\phi} \\
\dot{\theta} \\
\dot{\psi}
\end{array}\right]=\left[\begin{array}{ccc}
1 & \sin \phi \tan \theta & \cos \phi \tan \theta \\
0 & \cos \phi & -\sin \phi \\
0 & \sin \phi \sec \theta & \cos \phi \sec \theta
\end{array}\right]\left[\begin{array}{l}
p \\
q \\
r
\end{array}\right] .
$$

Assuming that the changes of roll and pitch angles are small, the transformation matrix between the angular velocities and the Euler angle rates is close to an identity matrix. Thus, the nonlinear system in (4) is linearized as

$$
\left\{\begin{array}{l}
\dot{\phi}=p \\
\dot{\theta}=q \\
\dot{\psi}=r
\end{array}\right.
$$

To facilitate the subsequent design procedures, (3) and (5) are simplified as the following nonlinear affine system:

$$
\left\{\begin{array}{l}
\dot{x}=A x+D f(x)+B u+W \Delta_{d}, \\
y=C x+F f_{s},
\end{array}\right.
$$

where $\quad x \in \mathbb{R}^{n}=[\phi, \theta, \psi, p, q, r]^{T}, y \in \mathbb{R}^{p}=[\phi, \theta, \psi]^{T}$, $u \in \mathbb{R}^{m}=\left[u_{\phi}, u_{\theta}, u_{\psi}\right]^{T}, f(x) \in \mathbb{R}^{s}=[q r, p r, p q]^{T}, \quad \Delta_{d} \in$ $\mathbb{R}^{r}, r$ represents the lumped model uncertainties and external disturbances, which are assumed to be unknown but bounded, and $f_{s} \in \mathbb{R}^{q}$ is an unknown vector which represents an additive bias resulting from sensor faults.

According to the above definitions, the known constant matrices in (6) can be described as

$$
\begin{aligned}
A & =\left[\begin{array}{llllll}
0 & 0 & 0 & 1 & 0 & 0 \\
0 & 0 & 0 & 0 & 1 & 0 \\
0 & 0 & 0 & 0 & 0 & 1 \\
0 & 0 & 0 & 0 & 0 & 0 \\
0 & 0 & 0 & 0 & 0 & 0 \\
0 & 0 & 0 & 0 & 0 & 0
\end{array}\right], \\
B & =\left[\begin{array}{cccc}
0 & 0 & 0 \\
0 & 0 & 0 \\
0 & 0 & 0 \\
J_{x}^{-1} & 0 & 0 \\
0 & J_{y}^{-1} & 0 \\
0 & 0 & J_{z}^{-1}
\end{array}\right],
\end{aligned}
$$

$$
\begin{aligned}
D & =\left[\begin{array}{cccc}
0 & 0 & 0 \\
0 & 0 & 0 \\
0 & 0 & 0 \\
\frac{J_{y}-J_{z}}{J_{x}} & 0 & 0 \\
& 0 & \frac{J_{z}-J_{x}}{J_{y}} & 0 \\
& & & \\
& 0 & & 0
\end{array}\right], \\
C & =\left[\begin{array}{llllll}
1 & 0 & 0 & 0 & 0 & 0 \\
0 & 1 & 0 & 0 & 0 & 0 \\
0 & 0 & 1 & 0 & 0 & 0
\end{array}\right] .
\end{aligned}
$$

\section{Active Fault-Tolerant Control Strategy}

3.1. Overview of the Proposed AFTC Scheme. A general structure of the developed AFTC scheme against sensor faults and disturbances is illustrated in Figure 2. The overall control system is constructed by a sensor fault diagnosis unit and a disturbance observer-based sliding-mode controller. The real-time information about sensor faults is provided by the fault diagnosis unit. Subsequently, the disturbance observer-based sliding-mode controller is proposed and integrated with the fault diagnosis unit to drive the quadrotor helicopter track the reference signals in the presence of sensor faults and disturbances.

\subsection{Sensor Fault Diagnosis}

Assumption 1. $\operatorname{rank}(\mathrm{CW})=\operatorname{rank}(\mathrm{W})$.

Assumption 2. The nonlinear term $f(x)$ is assumed to be known and Lipschitz about $x$ uniformly, i.e., $\forall x_{1}, x_{2} \in \mathbb{R}^{n}$,

$$
\left\|f\left(x_{1}\right)-f\left(x_{2}\right)\right\| \leq\left\|\mathscr{L}_{f} x_{1}-x_{2}\right\|,
$$

where $\mathscr{L}_{f}$ is the Lipschitz constant and is assumed to be unknown.

Assumption 3. The considered sensor faults $f_{s}$ and their derivatives $\dot{f}_{s}$ are bounded and defined by

$$
\varepsilon_{s}=\sup _{t>0}\left\|f_{s}\right\| \text {, and } \varepsilon_{s s}=\sup _{t>0}\left\|\dot{f}_{s}\right\| \text {. }
$$

Lemma 1. Under Assumption 1, there exist linear transformations of state and output: 


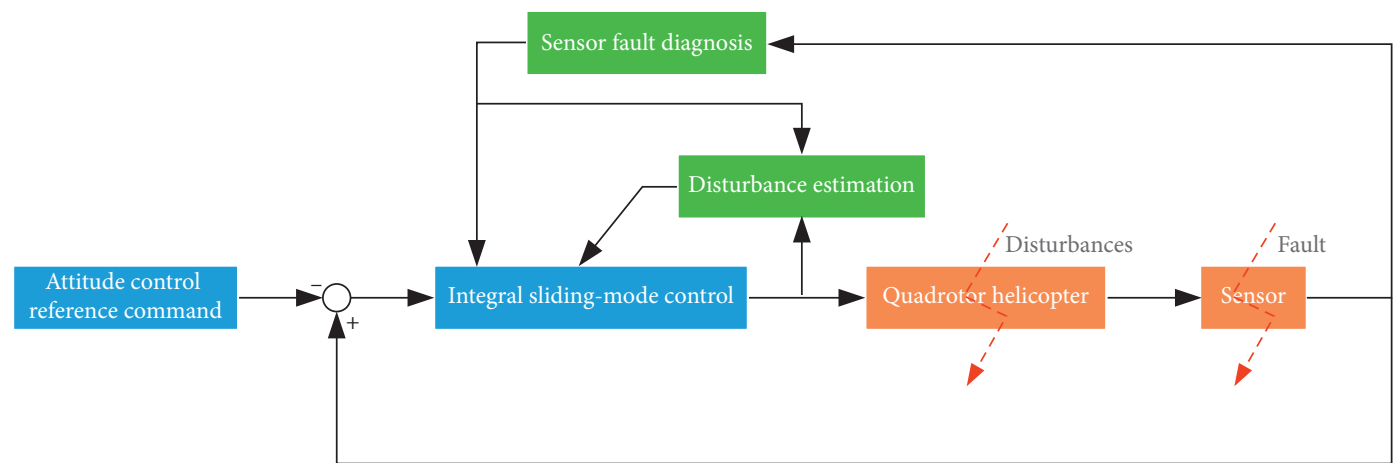

FIgURE 2: The schematic of the proposed AFTC strategy in the presence of sensor faults and external disturbances.

$$
\begin{aligned}
& z=T x=\left[\begin{array}{l}
z_{1} \\
z_{2}
\end{array}\right], \\
& h=S y=\left[\begin{array}{l}
h_{1} \\
h_{2}
\end{array}\right],
\end{aligned}
$$

such that the system matrices become

$$
\begin{aligned}
\mathrm{TAT}^{-1} & =\left[\begin{array}{ll}
A_{1} & A_{2} \\
A_{3} & A_{4}
\end{array}\right], \\
\mathrm{TB} & =\left[\begin{array}{l}
B_{1} \\
B_{2}
\end{array}\right], \\
\mathrm{TD} & =\left[\begin{array}{l}
D_{1} \\
D_{2}
\end{array}\right], \\
\mathrm{SCT}^{-1} & =\left[\begin{array}{cc}
C_{1} & 0 \\
0 & C_{4}
\end{array}\right], \\
\mathrm{SF} & =\left[\begin{array}{c}
0 \\
F_{2}
\end{array}\right], \\
\mathrm{TW} & =\left[\begin{array}{c}
W_{1} \\
0
\end{array}\right],
\end{aligned}
$$

where $T \in \mathbb{R}^{n \times n}, S \in \mathbb{R}^{p \times p}, z_{1} \in \mathbb{R}^{r}, z_{2} \in \mathbb{R}^{n-r}, \quad h_{1} \in \mathbb{R}^{r}$, $h_{2} \in \mathbb{R}^{p-r}, \quad A_{1} \in \mathbb{R}^{r \times r}, \quad A_{4} \in \mathbb{R}^{(n-r) \times(n-r)}, \quad B_{1} \in \mathbb{R}^{r \times m}$, $D_{1} \in \mathbb{R}^{r \times s}, C_{1} \in \mathbb{R}^{r \times r}, C_{4} \in \mathbb{R}^{(p-r) \times(n-r)}, F_{2} \in \mathbb{R}^{(p-r) \times q}$, and $W_{1} \in \mathbb{R}^{r \times r}$.

By applying both transformation matrices $T$ and $S$, original system (6) can be converted into the following two subsystems as

$$
\left\{\begin{array}{l}
\dot{z}_{1}=A_{1} z_{1}+A_{2} z_{2}+D_{1} f\left(T^{-1} z\right)+B_{1} u+W_{1} \Delta_{d} \\
h_{1}=C_{1} z_{1}
\end{array}\right.
$$

$$
\left\{\begin{array}{l}
\dot{z}_{2}=A_{3} z_{1}+A_{4} z_{2}+D_{2} f\left(T^{-1} z\right)+B_{2} u, \\
h_{2}=C_{4} z_{2}+F_{2} f_{s} .
\end{array}\right.
$$

After coordinate transformations, one can observe that subsystem (12) is only sensitive to lumped disturbances, and subsystem (13) is free from lumped disturbances but subject to sensor faults. In order to estimate $f_{s}$, the sensor faults in (13) are treated as actuator faults by defining a new state $z_{3}=\int_{0}^{t} h_{2}(\tau) \mathrm{d} \tau$. In this case, an augmented system with the state vector $\bar{z}=\left[z_{2}, z_{3}\right]^{T}$ can be given as

$$
\left\{\begin{array}{l}
\dot{\bar{z}}=\bar{A} \bar{z}+\bar{A}_{3} z_{1}+\bar{D}_{2} f\left(T^{-1} z\right)+\bar{B} u+\bar{F} f_{s}, \\
h_{3}=\bar{C} \bar{z},
\end{array}\right.
$$

where $\quad \bar{z} \in \mathbb{R}^{n+p-2 r}, \quad h_{3}=z_{3} \in \mathbb{R}^{p-r}, \quad \bar{A}=\left[\begin{array}{ll}A_{4} & 0 \\ C_{4} & 0\end{array}\right] \epsilon$

$\mathbb{R}^{(n+p-2 r) \times(n+p-2 r)}, \quad \bar{A}_{3}=\left[\begin{array}{c}A_{3} \\ 0\end{array}\right] \in \mathbb{R}^{(n+p-2 r) \times r}, \quad \bar{D}_{2}=\left[\begin{array}{c}D_{2} \\ 0\end{array}\right]$

$\in \mathbb{R}^{(n+p-2 r) \times s}, \quad \bar{B}=\left[\begin{array}{c}B_{2} \\ 0\end{array}\right] \in \mathbb{R}^{(n+p-2 r) \times m}, \quad \bar{F}=\left[\begin{array}{c}0 \\ F_{2}\end{array}\right] \epsilon$

$\mathbb{R}^{(n+p-2 r) \times q}$, and $\bar{C}=\left[\begin{array}{ll}0 & I_{p-r}\end{array}\right] \in \mathbb{R}^{(p-r) \times(n+p-2 r)}$.

Moreover, since $z_{2}$ is used in subsystem (12), with the new state vector $\bar{z}$, subsystem (12) can be accordingly rewritten as

$$
\left\{\begin{array}{l}
\dot{z}_{1}=A_{1} z_{1}+\bar{A}_{2} \bar{z}+D_{1} f\left(T^{-1} z\right)+B_{1} u+W_{1} \Delta_{d} \\
h_{1}=C_{1} z_{1}
\end{array}\right.
$$

where $\bar{A}_{2}=\left[\begin{array}{ll}A_{2} & 0\end{array}\right] \in \mathbb{R}^{r \times(n+p-2 r)}$.

Therefore, for subsystems (15) and (14), two sliding mode observers are designed as follows:

$$
\left\{\begin{array}{l}
\dot{\hat{z}}_{1}=A_{1} \widehat{z}_{1}+\bar{A}_{2} \widehat{\bar{z}}+B_{1} u+\frac{1}{2} \widehat{k}_{1} P_{1} C_{1}^{-1}\left(h_{1}-\widehat{h}_{1}\right)+D_{1} f\left(T^{-1} \widehat{z}\right)+\left(A_{1}-A_{1}^{s}\right) C_{1}^{-1}\left(h_{1}-\widehat{h}_{1}\right)+v_{1}, \\
\widehat{h}_{1}=C_{1} \widehat{z}_{1},
\end{array}\right.
$$




$$
\left\{\begin{array}{l}
\dot{\vec{z}}=\bar{A} \widehat{z}+\bar{A}_{3} C_{1}^{-1} h_{1}+\bar{D}_{2} f\left(T^{-1} \widehat{z}\right)+\bar{B} u+\bar{L}\left(h_{3}-\widehat{h}_{3}\right)+\frac{1}{2} \widehat{k}_{2} \bar{D}_{2} \bar{H}\left(h_{3}-\widehat{h}_{3}\right)+\bar{F} v_{2}, \\
\widehat{h}_{3}=\bar{C} \bar{z}
\end{array}\right.
$$

where $\widehat{z}=\left[\left(C_{1}^{-1} S_{1} y\right)^{T},\left(\left[I_{n-r} 0\right] \widehat{z}\right)^{T}\right]^{T}, A_{1}^{s} \in \mathbb{R}^{r \times r}$ is a stable matrix that needs to be determined, $P_{1}$ is the Lyapunov matrix of $A_{1}^{s}, \bar{L}=\left[\bar{L}_{1} \bar{L}_{2}\right]^{T} \in \mathbb{R}^{(n+p-2 r) \times(p-r)}$ is the observer gain matrix with $\bar{L}_{1} \in \mathbb{R}^{(n-r) \times(p-r)}$, and $\bar{H} \in \mathbb{R}^{j \times(p-r)}$.

The discontinuous output error injection term $v_{1}$, which is used to eliminate the effects of disturbances on state estimation, is designed as

$$
v_{1}= \begin{cases}\left(\left\|W_{1}\right\| \xi+\epsilon_{1}\right) \frac{P_{1}\left(C_{1}^{-1} h_{1}-\widehat{z}_{1}\right)}{\left\|P_{1}\left(C_{1}^{-1} h_{1}-\widehat{z}_{1}\right)\right\|}, & \text { if } C_{1}^{-1} h_{1} \neq \widehat{z}_{1}, \\ 0, & \text { otherwise, }\end{cases}
$$

where $\epsilon_{1}$ is a positive scalar to be determined.

Similarly, the discontinuous output error injection term $v_{2}$ can be designed as

$$
v_{2}= \begin{cases}\left(\epsilon_{s}+\epsilon_{2}\right) \frac{F_{0}\left(h_{3}-\widehat{h}_{3}\right)}{\left\|F_{0}\left(h_{3}-\widehat{h}_{3}\right)\right\|,} & \text { if } h_{3} \neq \widehat{h}_{3} \\ 0, & \text { otherwise }\end{cases}
$$

where $F_{0} \in \mathbb{R}^{q \times(p-r)}$ is a matrix and $\epsilon_{2}$ is a positive scalar that needs to be determined.

In addition, $\widehat{k}_{1}$ and $\dot{\hat{k}}_{2}$ are determined by the following adaptation laws as

$$
\begin{aligned}
& \dot{\hat{k}}_{1}=l_{k_{1}} P_{1}\left(C_{1}^{-1} h_{1}-\widehat{z}_{1}\right)^{2}, \\
& \dot{\widehat{k}}_{2}=l_{k_{2}} \bar{H}\left(h_{3}-\widehat{h}_{3}\right)^{2},
\end{aligned}
$$

where $l_{k_{1}}$ and $l_{k_{2}}$ are positive constants.

Then, by defining the state estimation errors as $\widetilde{z}_{1}=$ $z_{1}-\widehat{z}_{1}$ and $\widetilde{\bar{z}}=\bar{z}-\widehat{\bar{z}}$, the corresponding sliding surface can be constructed as

$$
\sigma=\left\{\left(\widetilde{z}_{1}, \tilde{\bar{z}}\right): \widetilde{z}_{1}=0, C \widetilde{\bar{z}}=0\right\} .
$$

Accordingly, the state estimation error dynamics can be obtained as follows:

$$
\begin{aligned}
& \dot{\tilde{z}}_{1}=A_{1}^{s} \widetilde{z}_{1}+\bar{A}_{2} \widetilde{\bar{z}}+D_{1}\left(f\left(T^{-1} z\right)-f\left(T^{-1} \widehat{z}\right)\right)-\frac{1}{2} \widehat{k}_{1} P_{1} \widetilde{z}_{1}+W_{1} \Delta_{d}-v_{1}, \\
& \dot{\bar{z}}=(\bar{A}-\bar{L} \bar{C}) \overline{\bar{z}}+\bar{D}_{2}\left(f\left(T^{-1} z\right)-f\left(T^{-1} \widehat{z}\right)^{\prime}\right)-\frac{1}{2} \widehat{k}_{2} \bar{D}_{2} \bar{H} \bar{C} \widetilde{\bar{z}}+\bar{F}\left(f_{s}-v_{2}\right) .
\end{aligned}
$$

Considering the fact that, after reaching the constructed sliding surface, the desired sliding motion needs to be maintained thereafter, namely, $\bar{C} \widetilde{\bar{z}}=0$, which results in that

$$
\bar{C}(\bar{A}-\bar{L} \bar{C}) \widetilde{z}+\bar{C}_{2}\left(f\left(T^{-1} z\right)-f\left(T^{-1} \widehat{z}\right)\right)-\frac{1}{2} \widehat{k}_{2} \bar{C}_{2} \overline{H C} \widetilde{\bar{z}}+\bar{C} \bar{F}\left(f_{s}-v_{2 e q}\right)=0,
$$

where $v_{2 e q}$ denotes the equivalent output error injection signal, which can be approximated as

$$
v_{2 e q}=\left(\epsilon_{s}+\epsilon_{2}\right) \frac{F_{0}\left(h_{3}-\widehat{h}_{3}\right)}{\left\|F_{0}\left(h_{3}-\widehat{h}_{3}\right)\right\|+\delta},
$$

with $\delta$ representing a small positive scalar for reducing the chattering effect.

In this sense, according to (25), the sensor fault can be approximated as

$$
\widehat{f}_{s} \approx\left(\epsilon_{s}+\epsilon_{2}\right) \frac{F_{0}\left(h_{3}-\widehat{h}_{3}\right)}{\left\|F_{0}\left(h_{3}-\widehat{h}_{3}\right)\right\|+\delta} .
$$

Theorem 1. Given a nonlinear affine system (6) with Assumptions 1-3 and the proposed observers (16) and (17), by employing adaptation schemes (20) and (21), state estimation error dynamics (23) and (24) can be driven to the defined sliding surface given by (22) if the gains $\epsilon_{1}$ and $\epsilon_{2}$ satisfy that 


$$
\begin{aligned}
& \epsilon_{1} \geq\left\|\bar{A}_{2}\right\|\|\tilde{\bar{z}}\|+\mathscr{L}_{f}\left\|T^{-1}\right\| D_{1}\|\tilde{z}\|+\eta_{1}, \\
& \epsilon_{2} \geq \mathscr{L}_{f}\left\|T^{-1}\right\| \bar{D}_{2} \frac{\|\tilde{z}\|}{\|\bar{F}\|}+\eta_{2},
\end{aligned}
$$

where $\eta_{1}$ and $\eta_{2}$ are positive scalars.
Proof of Theorem 1. Consider the following Lyapunov candidate functions:

$$
\begin{aligned}
& V_{1}=\widetilde{z}_{1}^{T} P_{1} \widetilde{z}_{1}, \\
& V_{2}=\widetilde{z}^{T} \bar{P} \widetilde{z} .
\end{aligned}
$$

Then, by substituting equations (23) and (24), the time derivative of $V_{1}$ and $V_{2}$ can be, respectively, calculated as

$$
\begin{aligned}
& \dot{V}_{1}=\widetilde{z}_{1}^{T}\left(A_{1}^{s T} P_{1}+P_{1} A_{1}^{s}\right) \widetilde{z}_{1}+2 \widetilde{z}_{1}^{T} P_{1} \overline{A_{2}} \widetilde{\bar{z}}+2 \widetilde{z}_{1}^{T} P_{1} W_{1} \Delta_{d}-\widehat{k}_{1}\left\|P_{1} \widetilde{z}_{1}^{2}\right\|+2 \widetilde{z}_{1}^{T} P_{1} D_{1}\left(f\left(T^{-1} z\right)-f\left(T^{-1} \widehat{z}\right)\right)-2 \widetilde{z}_{1}^{T} P_{1} v_{1}, \\
& \dot{V}_{2}=\widetilde{z}^{T}\left((\bar{A}-\overline{L C})^{T} \bar{P}+\bar{P}(\bar{A}-\overline{L C})\right)^{\underline{L}}-\widehat{k}_{2} \widetilde{z}^{T} \overline{P D_{2}} \overline{H C} \overline{\bar{z}}+2 \widetilde{z}^{T} \overline{P D}_{2}\left(f\left(T^{-1} z\right)-f\left(T^{-1} \widehat{z}\right)\right)+2 \widetilde{z}^{T} \overline{P F}\left(f_{s}-v_{2}\right) .
\end{aligned}
$$

Since $P_{1}=P_{1}{ }^{T}>0$ and $A_{1}^{s}<0$, (31) and (32) can be rewritten as follows:

$$
\begin{aligned}
\dot{V}_{1} & \leq 2 \widetilde{z}_{1}^{T} P_{1} \bar{A}_{2} \widetilde{\tilde{z}}+2 \widetilde{z}_{1}^{T} P_{1} D_{1}\left(f\left(T^{-1} z\right)-f\left(T^{-1} \widehat{z}\right)\right)+2 \widetilde{z}_{1}^{T} P_{1} W_{1} \Delta_{d}-2 \widetilde{z}_{1}^{T} P_{1} v_{1} \\
& =2 \widetilde{z}_{1}^{T} P_{1}\left(\bar{A}_{2} \tilde{\bar{z}}+D_{1}\left(f\left(T^{-1} z\right)-f\left(T^{-1} \widehat{z}\right)\right)+W_{1} \Delta_{d}-v_{1}\right) \leq 2 P_{1} \widetilde{z}_{1}\left(\bar{A}_{2}\|\widetilde{z}\|+\mathscr{L}_{f} T^{-1} D_{1}\|\widetilde{z}\|-\epsilon_{1}\right), \\
\dot{V}_{2} & \leq 2 \widetilde{z}^{T} \overline{P D}_{2}\left(f\left(T^{-1} z\right)-f\left(T^{-1} \widehat{z}\right)\right)-\widehat{k}_{2} \widetilde{\bar{z}}^{T} \overline{P D}_{2} \overline{H C} \widetilde{\bar{z}}+2 \widetilde{z}^{T} \overline{P F}\left(f_{s}-v_{2}\right) \\
& \leq 2\|P \widetilde{z}\|\left(\mathscr{L}_{f} T^{-1} D_{2}\|\tilde{z}\|-\|F\| \epsilon_{2}\right) .
\end{aligned}
$$

Finally, substituting (28) and (29) into (33) and (34) leads to that

$$
\begin{aligned}
& \dot{V}_{1} \leq-2 \eta_{1} P_{1} \widetilde{z}_{1}, \\
& \dot{V}_{2} \leq-2 \eta_{2}\|\bar{P} \widetilde{z}\| .
\end{aligned}
$$

Therefore, the desired sliding motion can be achieved and maintained.

3.3. Integral Sliding-Mode Control Design. The design of a sliding mode controller is typically composed of two steps. The first step features the construction of a sliding surface, on which the system performance can be maintained as expected. The second step is concerned with the selection of an appropriate control law to drive the sliding variable reach the designed sliding surface and thereafter keep the sliding motion within the close neighborhood of the sliding surface. However, during the reaching phase, the insensitivity of the sliding mode controller cannot be ensured. One way to solve this problem is to employ the integral sliding-mode control scheme, such that the robustness of the system can be guaranteed throughout the entire response of the system starting from the initial time instant [35].

In order to facilitate the integral sliding-mode control design, the nonlinear system described in (6) can be rewritten in the following integral chain format as

$$
\left\{\begin{array}{l}
\dot{x}_{1}=x_{2}, \\
\dot{x}_{2}=f\left(x_{2}\right)+B_{u} u+d,
\end{array}\right.
$$

where $x_{1}=[\phi, \theta, \psi]^{T}$ and $x_{2}=[p, q, r]^{T}$.

By denoting $x_{1}^{d}$ as the desired trajectory of the quadrotor helicopter, the corresponding tracking error vector can be defined as

$$
x_{1}^{e}=x_{1}-x_{1}^{d}=\left[\begin{array}{c}
\phi-\phi_{d} \\
\theta-\theta_{d} \\
\psi-\psi_{d}
\end{array}\right] .
$$

Then, with this tracking error vector (37), the integral sliding surface for the controlled system is defined as follows:

$$
S=\dot{x}_{1}^{e}+k_{c 2} x_{1}^{e}+k_{c 1} \int_{t_{0}}^{t} x_{1}^{e}(\tau) \mathrm{d} \tau-k_{c 2} x_{1}^{e}\left(t_{0}\right)-\dot{x}_{1}^{e}\left(t_{0}\right),
$$

where $t_{0}$ is the initial time instant and the diagonal matrices $k_{c 1}$ and $k_{c 2}$ represent the design parameters.

From this definition, one can observe that the integral term renders the sliding motion occur at the initial time instant $t_{0}$. Accordingly, the system trajectory under integralsliding mode control starts from the designed sliding surface, and the reaching phase is eliminated compared to conventional sliding mode control.

The continuous sliding-mode control part is designed by equalizing $\dot{S}=0$ as 


$$
u_{0}=B_{u}^{-1}\left(\dot{x}_{1}^{d}-k_{c 2} \dot{x}_{1}^{e}-k_{c 1} x_{1}^{e}-f\left(x_{2}\right)\right) .
$$

The discontinuous control part to reject disturbances is designed as

$$
u_{1}=-B_{u}^{-1} k_{c 3} \operatorname{sat}(S),
$$

where $k_{c 3}$ is a diagonal matrix constructed with positive high gains that make the designed sliding surface attractive, and the sat function is defined as [36]:

$$
\operatorname{sat}(S)= \begin{cases}\operatorname{sign}(S), & \text { if }\|S\|>\Phi, \\ \frac{S}{\Phi}, & \text { if }\|S\| \leq \Phi,\end{cases}
$$

with $\Phi$ representing the boundary layer thickness.

However, in order to account for the unknown disturbances, the discontinuous control gain $k_{c 3}$ needs to be chosen greater than the upper bound of the unknown disturbances, which may lead to unexpected control chattering. Therefore, a nonlinear disturbance observer needs to be designed and synthesized with the designed sliding-mode control to reduce the discontinuous control gain.

\subsection{Nonlinear Disturbance Observer Design and Synthesis with Sliding Mode Control}

Assumption 4. The disturbances in system (36) are bounded and defined by $D_{d}=\sup _{t>0}\|d\|$.

Assumption 5. The lumped disturbances can be represented by the following exogenous system $[37,38]$ :

$$
\left\{\begin{array}{l}
\dot{\zeta}=A_{d} \zeta, \\
d=C_{d} \zeta .
\end{array}\right.
$$

To estimate the unknown disturbances, a nonlinear disturbance observer is designed as

$$
\begin{aligned}
& \dot{\bar{z}}=\left[A_{d}-\lambda C_{d}\right] z+A_{d} \mu-\lambda\left[C \mu+f\left(x_{2}\right)+B_{u} u\right] \\
& \widehat{\zeta}=z+\mu, \\
& \widehat{d}=C_{d} \widehat{\zeta} .
\end{aligned}
$$

With the estimated disturbances $\widehat{d}$, the disturbance estimation error is defined as

$$
d_{e}=d-\widehat{d}
$$

Assumption 6. The disturbance estimation error is bounded by $D_{e}=\sup _{t>0}\left\|d_{e}\right\|$.

By recalling the sliding mode control derived in (39) and (40), with the estimated sensor faults and disturbances, the composite control law can be constructed as follows:

$$
u=B_{u}^{-1}\left(\ddot{\hat{x}}_{1}^{d}-k_{c 2} \dot{\hat{x}}_{1}^{e}-k_{c 1} \hat{x}_{1}^{e}-f\left(x_{2}\right)-\widehat{d}\right)-B_{u}^{-1} k_{c 3} \operatorname{sat}(S) \text {. }
$$

Theorem 2. Consider a nonlinear affine system (36) with exogenous disturbances (42). Given the designed integral sliding surface (38), by employing the disturbance estimation (43) and the feedback control law (45), the system tracking performance can be guaranteed as expected in the presence of unknown disturbances with the discontinuous control gain chosen as $k_{c 3} \geq \eta+D_{e}$.

Proof of Theorem 2. To prove Theorem 2, a nonnegative Lyapunov function is defined as

$$
V_{3}=\frac{1}{2} S^{T} S \text {. }
$$

The time derivative of $V_{3}$ for $S \neq 0$ can be calculated as

$$
\begin{aligned}
\dot{V}_{3} & =S^{T} \dot{S}=S^{T}\left(f\left(x_{2}\right)+B_{u} B_{u}^{-1}\left(\ddot{\widetilde{x}}_{1}^{d}-k_{c 2} \dot{\hat{x}}_{1}^{e}-k_{c 1} \hat{x}_{1}^{e}-f\left(x_{2}\right)-\hat{d}-k_{c 3} \operatorname{sat}(S)\right)+d-\ddot{\widetilde{x}}_{1}^{d}+k_{c 2} \dot{\hat{x}}_{1}^{e}+k_{c 1} \hat{x}_{1}^{e}\right), \\
& =S^{T}\left(-k_{c 3} \operatorname{sat}(S)+d-\hat{d}\right) \leq S^{T}\left(-\left(\eta+D_{e}\right) \operatorname{sat}(S)+D_{e}\right) \leq-\eta\|S\| .
\end{aligned}
$$

Therefore, the stability of the controller system can be guaranteed with the designed control law (45) in the presence of unknown exogenous disturbances.

Remark 1. Since the unknown disturbances have been precisely estimated by the disturbance observer, the magnitude of the estimation error can be kept much smaller than that of the disturbance. Thus, compared to conventional sliding-mode control, the value of the discontinuous control gain is significantly reduced, which can alleviate the control chattering problem to some extent.

\section{Simulation Tests and Discussion}

In this section, the proposed AFTC strategy against sensor faults and external disturbances will be validated. To achieve an effective evaluation of the proposed AFTC strategy, three different faulty patterns are considered for the attitude control system of the quadrotor helicopter. In addition, for comparison, the tracking performance of a conventional sliding-mode control is also investigated to demonstrate the advantages of the proposed control strategy.

It can be observed from Figures $3-5$ that during $20<t<40$ s and $50<t<70$ s, all the attitude measurement systems fail simultaneously. By using the proposed sensor 


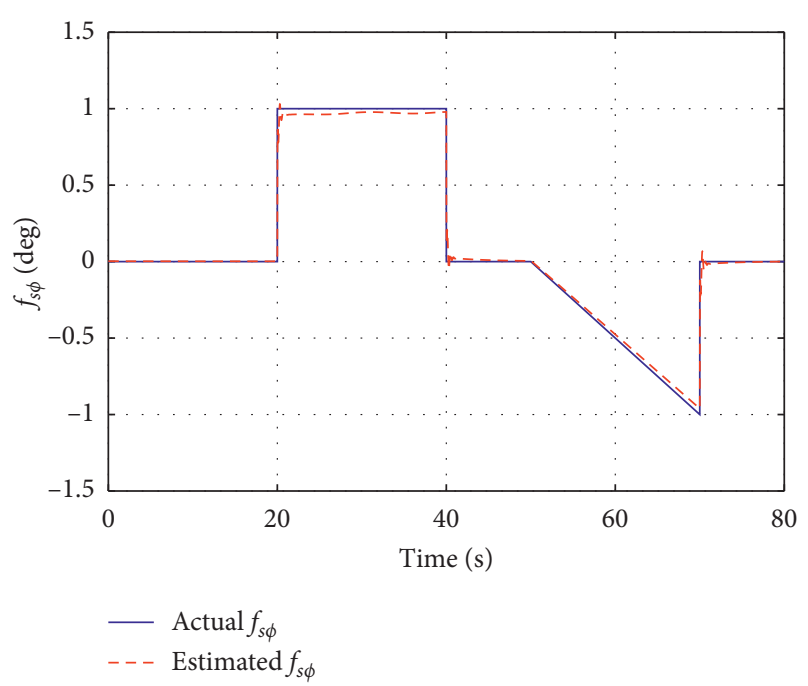

Figure 3: Performance of the $f_{s \phi}$ sensor fault estimation in the presence of external disturbances.

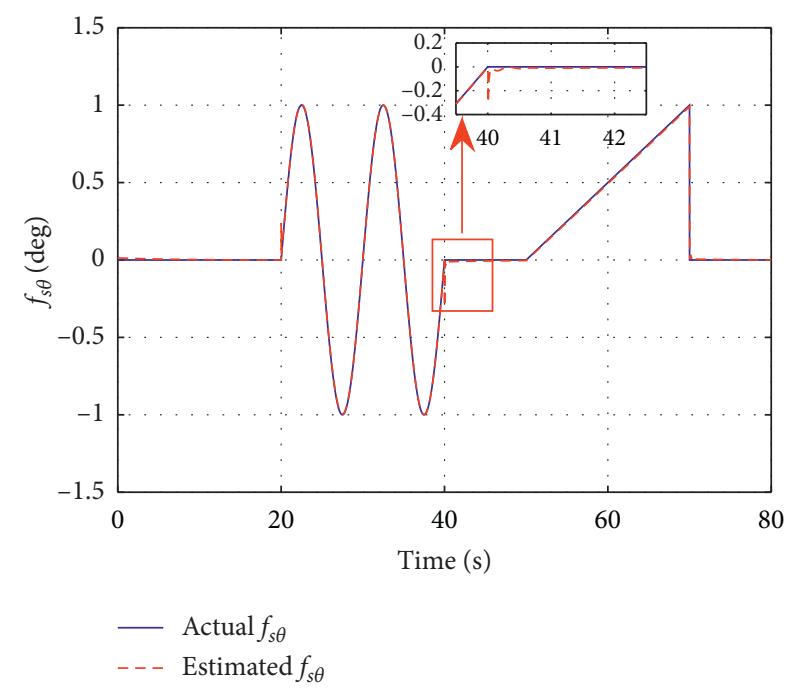

Figure 4: Performance of the $f_{s \theta}$ sensor fault estimation in the presence of external disturbances.

fault estimation scheme, the sensor faults can be accurately estimated, and the unbiased attitude estimation is provided for the feedback control. For comparison, the tracking performance of the compared conventional sliding-mode control without the sensor fault diagnosis system is studied, which is shown as the dash-dotted red line in Figures 6-8. In this case, the attitude measurements are directly utilized by the feedback controller. Therefore, the attitude of the quadrotor helicopter cannot follow the reference commands when there exist sensor faults, and the response of the quadrotor helicopter is obviously influenced by the wrong sensor measurements. For example, as shown in Figure 6,

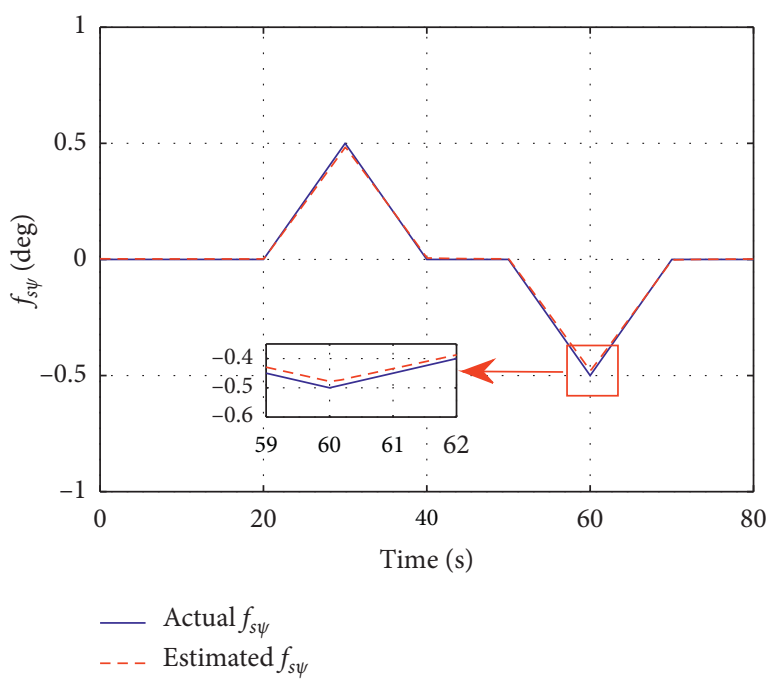

Figure 5: Performance of the $f_{s \psi}$ sensor fault estimation in the presence of external disturbances.

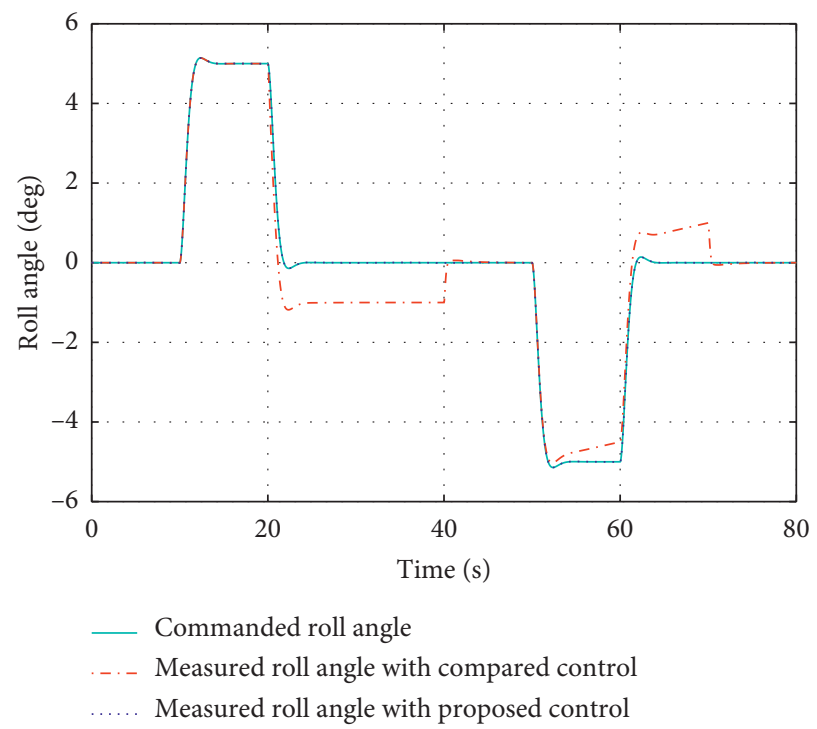

FIGURE 6: Comparison of roll tracking performance in the presence of sensor faults.

during $20<t<40$ s, the reference command for the roll angle is zero, but the response is -1 deg due to the occurrence of sensor fault. In contrast, with the sensor diagnosis system, the sensor faults can be accurately estimated, and the quadrotor helicopter can still follow the reference commands very well even in the presence of sensor faults as can be observed from the dashed blue line of Figures 6-8.

In order to further test the capability of the proposed control strategy against both sensor faults and external disturbances, two different scenarios are tested. In scenario 1 , in addition to the aforementioned sensor faults, at $5 \mathrm{~s}$, a 


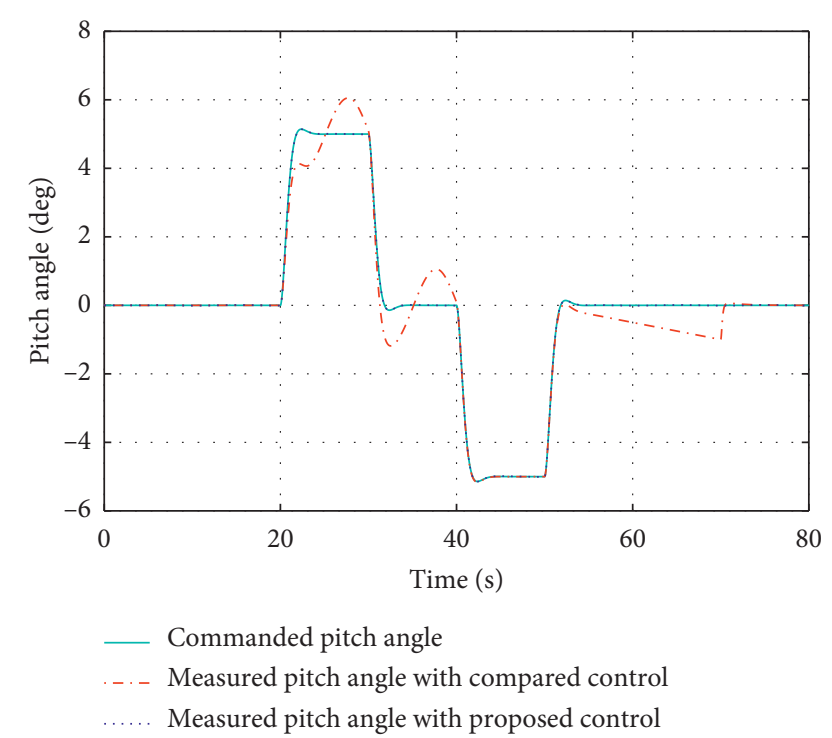

Figure 7: Comparison of pitch tracking performance in the presence of sensor faults.

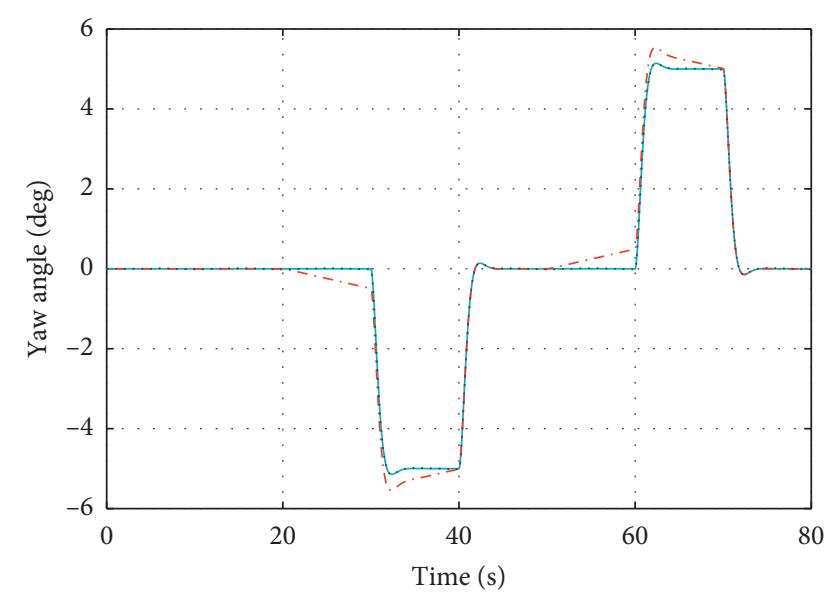

- Commanded yaw angle

- . - Measured yaw angle with compared control

.... Measured yaw angle with proposed control

FIGURE 8: Comparison of yaw tracking performance in the presence of sensor faults.

constant external disturbance with three different amplitudes is injected to roll, pitch, and yaw motions, respectively. The attitude tracking performances are demonstrated in Figures 9-11. It can be observed that, after the disturbance is injected to the roll, pitch, and yaw motion of the quadrotor helicopter at $5 \mathrm{~s}$, even if there is no sensor fault at that time, there still exist attitude tracking errors with the compared control, as shown in the dash-dotted red line. However, with

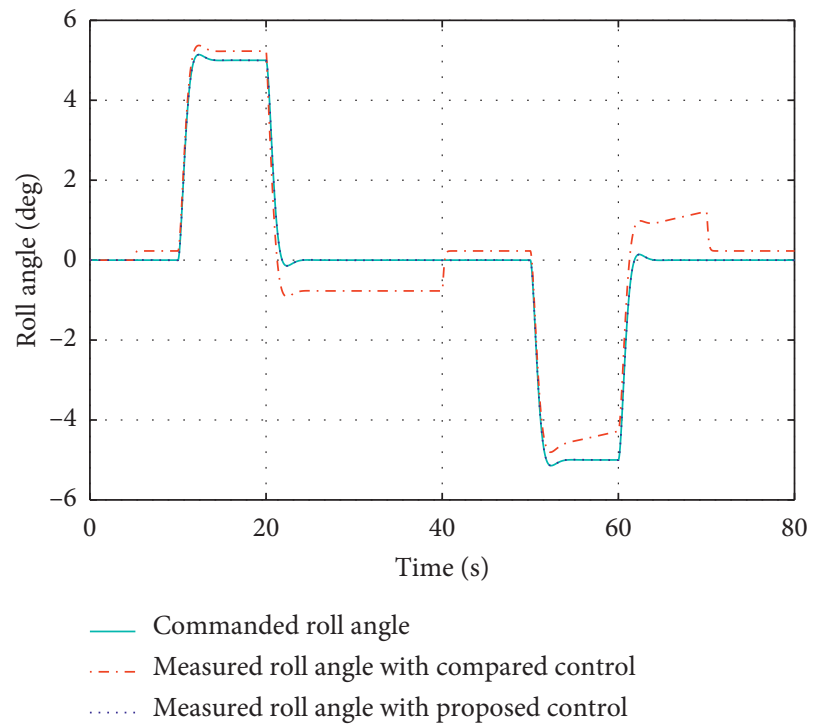

FIGURE 9: Comparison of roll tracking performance in the presence of both sensor faults and constant external disturbances.

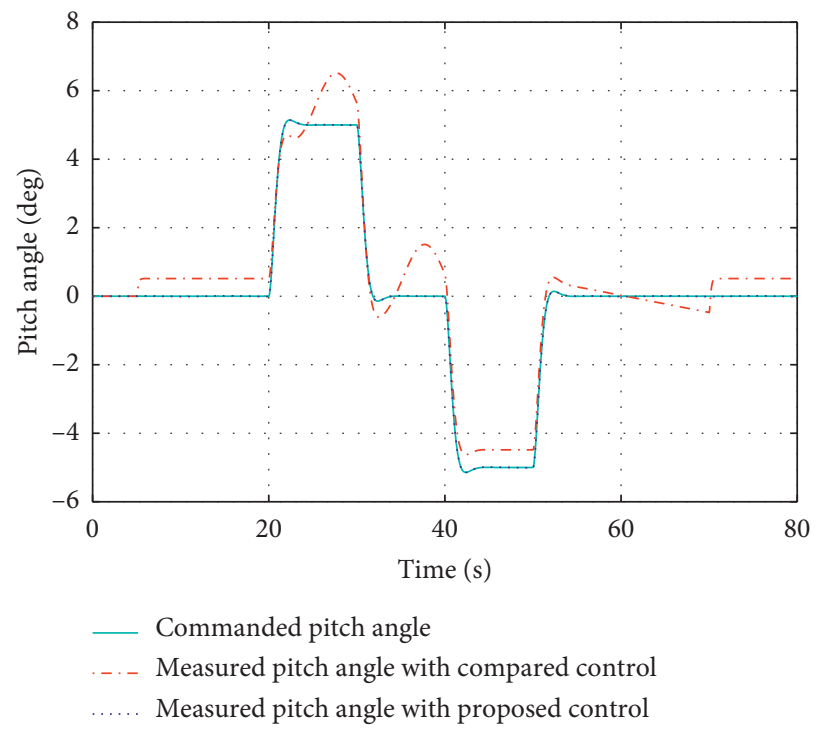

FIGURE 10: Comparison of pitch tracking performance in the presence of both sensor faults and constant external disturbances.

the proposed control, the injected external disturbances can be estimated and compensated accordingly. In this case, the desired attitude tracking performance can be maintained even in the presence of both sensor faults and constant external disturbances, as shown in the dashed blue line.

In scenario 2, a time-varying disturbance with three different amplitudes is injected to the quadrotor helicopter at $5 \mathrm{~s}$ to further validate the tracking performance of the 


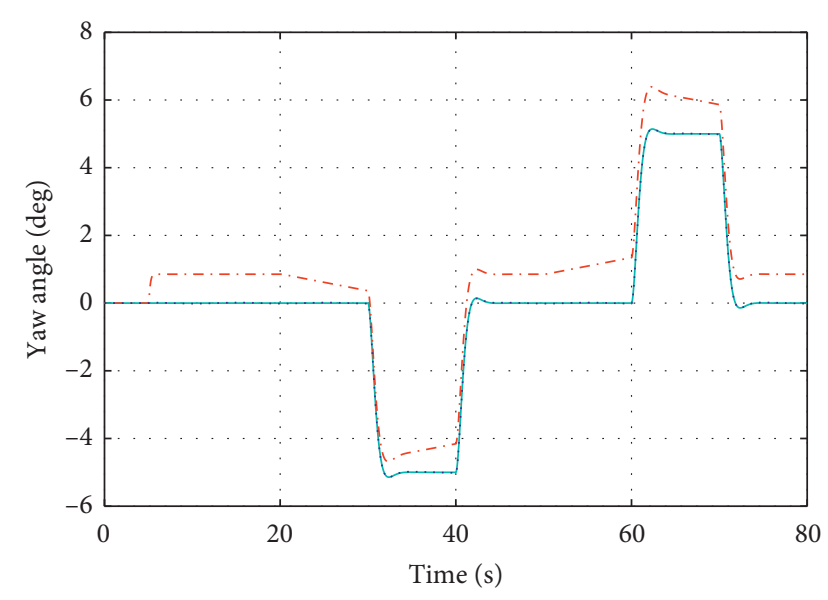

_ Commanded yaw angle

_. - Measured yaw angle with compared control

.... Measured yaw angle with proposed control

FIGURE 11: Comparison of yaw tracking performance in the presence of both sensor faults and constant external disturbances.

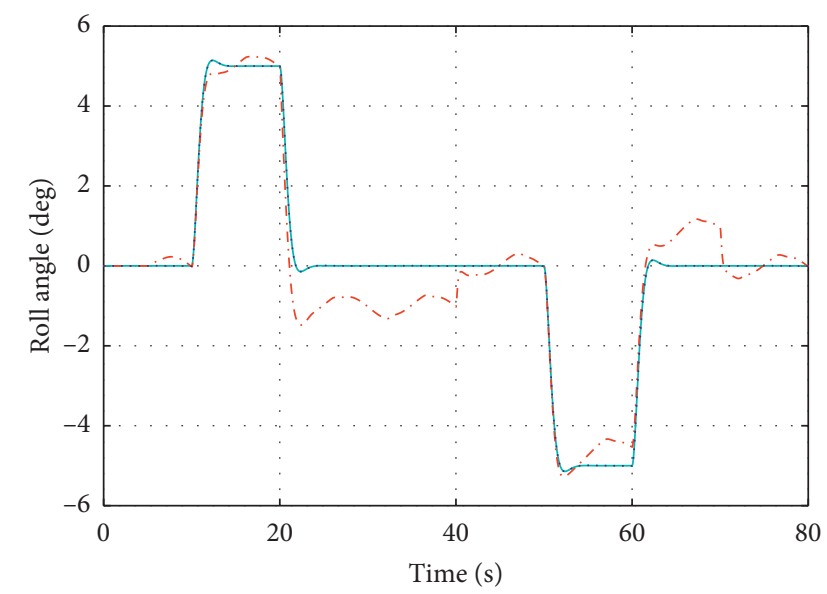

_ Commanded roll angle

- - Measured roll angle with compared control

.... Measured roll angle with proposed control

FIgURE 12: Comparison of roll tracking performance in the presence of both sensor faults and time-varying external disturbances.

proposed control strategy in the presence of both sensor faults and time-varying external disturbances. The corresponding attitude tracking performances are shown in Figures 12-14. Compared to scenario 1, the system tracking performance with the compared control is more affected. On the contrary, even in this situation, the proposed control strategy can still maintain a satisfactory system tracking performance, which confirms the effectiveness and superiority of the proposed control strategy.

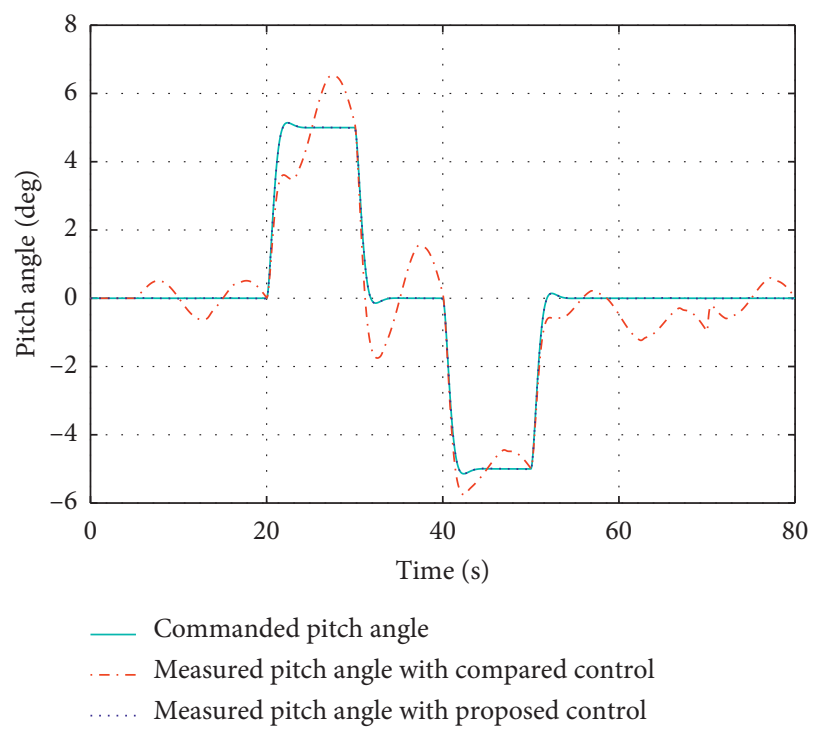

FIGURE 13: Comparison of pitch tracking performance in the presence of both sensor faults and time-varying external disturbances.

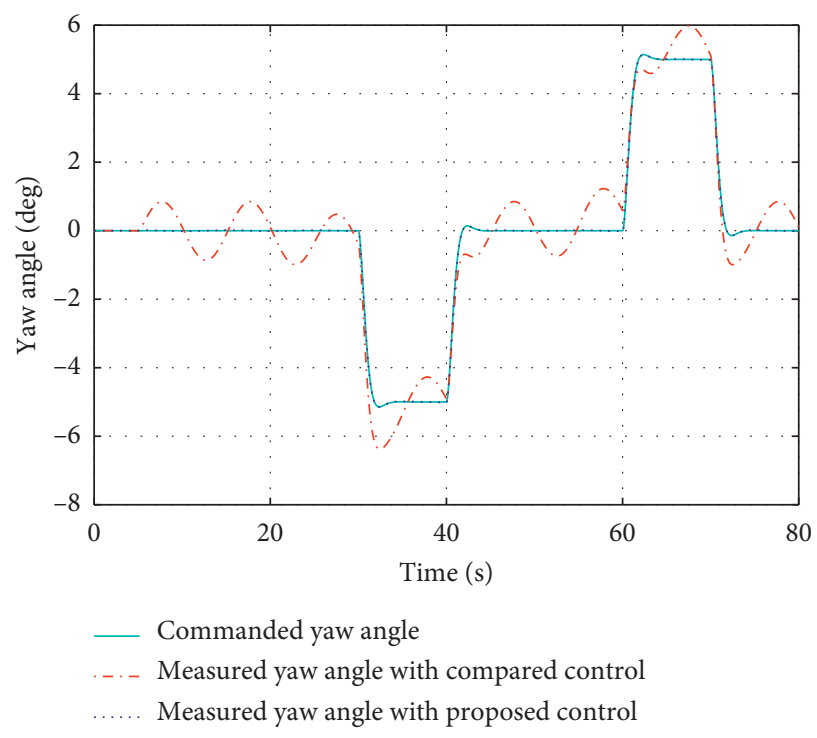

FIGURE 14: Comparison of yaw tracking performance in the presence of both sensor faults and time-varying external disturbances.

\section{Conclusions}

In this paper, an active fault-tolerant control strategy is proposed for a quadrotor helicopter against sensor faults and external disturbances. The proposed fault diagnosis scheme has the capability to accurately estimate the sensor faults and eliminate the effect of disturbances, which is then integrated with a robust controller in an active fashion to ensure the continuous operation of the quadrotor helicopter. For controlling the quadrotor helicopter, a disturbance observer 
is designed to effectively estimate the external disturbances and synthesized with the designed integral sliding-mode controller, which can help the baseline controller to avoid control chattering. In addition, the stability of the closedloop system is theoretically proved. In comparison with a conventional sliding-mode control, the demonstrated numerical simulation results show the effectiveness and advantages of the proposed active fault-tolerant control strategy under different faulty and uncertain scenarios.

\section{Data Availability}

The data used to support the findings of this study are included within this article.

\section{Conflicts of Interest}

The authors declare that there are no conflicts of interest.

\section{Acknowledgments}

This research work was partially supported by the Fundamental Research Funds for the Central Universities.

\section{References}

[1] T. Huang, D. Huang, Z. Wang, and A. Shah, "Robust tracking control of a quadrotor UAV based on adaptive sliding mode controller," Complexity, vol. 2019, Article ID 7931632, 15 pages, 2019.

[2] C. Li, Y. Zhang, and P. Li, "Full control of a quadrotor using parameter-scheduled backstepping method: implementation and experimental tests," Nonlinear Dynamics, vol. 89, no. 2, pp. 1259-1278, 2017.

[3] Y. Zhang, N. Xu, G. Zhu, L. Sun, S. Cao, and X. Zhang, "Adaptive robust dynamic surface integral sliding mode control for quadrotor UAVs under parametric uncertainties and external disturbances," Complexity, vol. 2020, Article ID 8879364, 20 pages, 2020.

[4] X. Yu, Y. Fu, and Y. Zhang, "Aircraft fault accommodation with consideration of actuator control authority and gyro availability," IEEE Transactions on Control Systems Technology, vol. 26, no. 4, pp. 1285-1299, 2018.

[5] R. Wang, Y. Li, H. Sun, Y. Zhang, and Y. Sun, "Performance analysis of switched control systems under common-source digital upsets modeled by MDHMM," Complexity, vol. 2018, Article ID 4329053, 12 pages, 2018.

[6] J. Zhang, A. K. Swain, and S. K. Nguang, "Robust sensor fault estimation scheme for satellite attitude control systems," Journal of the Franklin Institute, vol. 350, no. 9, pp. 2581-2604, 2013.

[7] R. Wang, C. Wang, H. Sun, Z. Chen, and Y. Sun, "Sensor fault signal reconstruction based on sliding mode observer for flight control systems," International Journal of Modelling, Identification and Control, vol. 32, no. 3-4, pp. 204-211, 2019.

[8] M. K. Jeerage, "Reliability analysis of fault-tolerant IMU architectures with redundant inertial sensors," IEEE Aerospace and Electronic Systems Magazine, vol. 5, no. 7, pp. 2328, 1990.

[9] Y. Zhong, W. Zhang, Y. Zhang, J. Zuo, and H. Zhan, "Sensor fault detection and diagnosis for an unmanned quadrotor helicopter," Journal of Intelligent \& Robotic Systems, vol. 96, no. 3-4, pp. 555-572, 2019.
[10] A. Matus-Vargas, G. Rodriguez-Gomez, and J. MartinezCarranza, "A monocular SLAM-based controller for multirotors with sensor faults under ground effect," Sensors, vol. 19, no. 22, p. 4948, 2019.

[11] Z. Zhang and B. Xiao, "Sensor fault reconstruction based on adaptive sliding mode observer for forklift fault-tolerant control system," Applied Sciences, vol. 10, no. 4, p. 1278, 2020.

[12] J. Tan, Y. Fan, P. Yan, C. Wang, and H. Feng, "Sliding mode fault tolerant control for unmanned aerial vehicle with sensor and actuator faults," Sensors, vol. 19, no. 3, p. 643, 2019.

[13] C. Edwards, S. K. Spurgeon, and R. J. Patton, "Sliding mode observers for fault detection and isolation," Automatica, vol. 36, no. 4, pp. 541-553, 2000.

[14] C. P. Tan and C. Edwards, "Sliding mode observers for detection and reconstruction of sensor faults," Automatica, vol. 38, no. 10, pp. 1815-1821, 2002.

[15] H. Alwi, C. Edwards, and C. P. Tan, "Sliding mode estimation schemes for incipient sensor faults," Automatica, vol. 45, no. 7, pp. 1679-1685, 2009.

[16] Y. Zhang and J. Jiang, "Bibliographical review on reconfigurable fault-tolerant control systems," Annual Reviews in Control, vol. 32, no. 2, pp. 229-252, 2008.

[17] Y. M. Zhang, A. Chamseddine, C. A. Rabbath et al., "Development of advanced FDD and FTC techniques with application to an unmanned quadrotor helicopter testbed," Journal of the Franklin Institute, vol. 350, no. 9, pp. 23962422, 2013.

[18] X. Fang, H. Fan, and L. Liu, "Robust finite-time tracking for uncertain linear systems with actuator faults," Complexity, vol. 2020, Article ID 2463790, 13 pages, 2020.

[19] P. Lyu, J. Lai, J. Liu, H. H. T. Liu, and Q. Zhang, "A thrust model aided fault diagnosis method for the altitude estimation of a quadrotor," IEEE Transactions on Aerospace and Electronic Systems, vol. 54, no. 2, pp. 1008-1019, 2018.

[20] F. Xu, J. Tan, X. Wang, V. Puig, B. Liang, and B. Yuan, "Mixed active/passive robust fault detection and isolation using settheoretic unknown input observers," IEEE Transactions on Automation Science and Engineering, vol. 15, no. 2, pp. 863871, 2018.

[21] Y. Al Younes, H. Noura, A. Rabhi, A. El Hajjaji, and N. Al Hussien, "Sensor fault detection and isolation in the quadrotor vehicle using nonlinear identity observer approach," in Proceedings of 2013 Conference on Control and Fault-Tolerant Systems, Nice, France, October 2013.

[22] J. Gao, Q. Zhang, and J. Chen, "EKF-based actuator fault detection and diagnosis method for tilt-rotor unmanned aerial vehicles," Mathematical Problems in Engineering, vol. 2020, Article ID 8019017, 12 pages, 2020.

[23] B. Wang, Y. Shen, and Y. Zhang, "Active fault-tolerant control for a quadrotor helicopter against actuator faults and model uncertainties," Aerospace Science and Technology, vol. 99, Article ID 105745, 2020.

[24] R. C. Avram, X. Zhang, and J. Muse, "Quadrotor accelerometer and gyroscope sensor fault diagnosis using nonlinear adaptive estimation methods," International Journal of Prognostics and Health Management, vol. 7, no. 1, pp. 1-10, 2016.

[25] R. C. Avram, X. Zhang, and J. Muse, "Quadrotor sensor fault diagnosis with experimental results," Journal of Intelligent \& Robotic Systems, vol. 86, no. 1, pp. 115-137, 2017.

[26] Y. Zhong, W. Zhang, and Y. M. Zhang, "Sensor fault diagnosis for unmanned quadrotor helicopter via adaptive two-stage extended Kalman filter," in Proceedings of 2017 International 
Conference on Sensing, Diagnostics, Prognostics, and Control (SDPC), pp. 493-498, Shanghai, China, August 2017.

[27] C. Zhu, C. Li, X. Chen, K. Zhang, X. Xin, and H. Wei, "Eventtriggered adaptive fault tolerant control for a class of uncertain nonlinear systems," Entropy, vol. 22, no. 6, p. 598, 2020.

[28] B. Wang and Y. Zhang, "An adaptive fault-tolerant sliding mode control allocation scheme for multirotor helicopter subject to simultaneous actuator faults," IEEE Transactions on Industrial Electronics, vol. 65, no. 5, pp. 4227-4236, 2018.

[29] F. Chen, W. Lei, K. Zhang, G. Tao, and B. Jiang, "A novel nonlinear resilient control for a quadrotor UAV via backstepping control and nonlinear disturbance observer," Nonlinear Dynamics, vol. 85, no. 2, pp. 1281-1295, 2016.

[30] N. P. Nguyen and S. K. Hong, "Active fault-tolerant control of a quadcopter against time-varying actuator faults and saturations using sliding mode backstepping approach," Applied Sciences, vol. 9, no. 19, p. 4010, 2019.

[31] B. Wang, X. Yu, L. Mu, and Y. Zhang, "A dual adaptive faulttolerant control for a quadrotor helicopter against actuator faults and model uncertainties without overestimation," Aerospace Science and Technology, vol. 99, p. 105744, 2020.

[32] R. Wang, L. Gao, C. Bai, and H. Sun, "U-Model-Based sliding mode controller design for quadrotor UAV control systems," Mathematical Problems in Engineering, vol. 2020, Article ID 4343214, 11 pages, 2020.

[33] F. R. Lopez-Estrada, J.-C. Ponsart, D. Theilliol, Y. M. Zhang, and C.-M. Astorga-Zaragoza, "LPV model-based tracking control and robust sensor fault diagnosis for a quadrotor UAV," Journal of Intelligent \& Robotic Systems, vol. 84, no. 14, pp. 163-177, 2016.

[34] L. Qin, X. He, R. Yan, and D. Zhou, "Active fault-tolerant control for a quadrotor with sensor faults," Journal of Intelligent \& Robotic Systems, vol. 88, no. 2-4, p. 449X67, 2017.

[35] V. Utkin and J. Shi, "Integral sliding mode in systems operating under uncertainty conditions," in Proceedings of 35th IEEE Conference on Decision and Control, pp. 4591-4596, Kobe, Japan, December 1996.

[36] J. J. Slotine and S. S. Sastry, "Tracking control of non-linear systems using sliding surfaces, with application to robot manipulators $\dagger$," International Journal of Control, vol. 38, no. 2, pp. 465-492, 1983.

[37] W.-H. Chen, "Disturbance observer based control for nonlinear systems," IEEE/ASME Transactions on Mechatronics, vol. 9, no. 4, pp. 706-710, 2004.

[38] W.-H. Chen, J. Yang, L. Guo, and S. H. Li, "Disturbanceobserver-based control and related methods-An overview," IEEE Transactions on Industrial Electronics, vol. 63, no. 2, pp. 1083-1095, 2015. 